\title{
Assessment of Harmonic Contribution of a Photovoltaic Installation Based on Field Measurements
}

\author{
Morteza Pourarab, Jan Meyer, Robert Stiegler \\ Institute of Electrical Power Systems and High Voltage Engineering \\ Technische Universitaet Dresden \\ Mommsenstraße 10, 01062 Dresden (Germany) \\ e-mail: morteza.pourarab@tu-dresden.de
}

\begin{abstract}
.
Quantifying harmonic contribution of customer installations to the voltage distortion in the network is still one of the main challenges of utilities with respect to power quality, particularly when integrating large number of renewable energy sources. This paper discusses different aspect of evaluating the low-order harmonic emission of a $100 \mathrm{~kW}$ photovoltaic (PV) installation consisting of 16 single-phase inverters. Based on field measurements of one week the harmonic currents of the installation are analyzed with respect to different operating conditions. The measured harmonic currents are compared with emission values provided by the manufacturer as well as emission limits specified in standards. Finally, different methods for the determination of the contribution of the PV installation to the voltage distortion in the network are compared.
\end{abstract}

\section{Keywords}

Harmonics, harmonic phase angle, harmonic contribution, PV installation, summation exponent.

\section{Introduction}

One of the main challenges when connecting photovoltaic systems is to quantify the harmonic contribution of such systems to the distortion in the distribution network. Basic guidelines for the calculation and evaluation of the harmonic emission limits for the connection of disturbing installations to LV networks are provided e.g. in the IEC TR 61000-3-14 [1]. In Germany, a country-specific rule, the VDE AR-N 4105 is applied for emission limit calculation [2].

In literature, several papers on harmonic emission of PV installations can be found. They majorly address harmonic emission limits, the impact of harmonic phase angle on emission or introduce new assessment indices [e.g. 3,4]. However, the identification of the harmonic contribution of a renewable installation to the supply voltage distortion is still an open issue.

Several methods exist for the assessment of the customer's share in harmonic distortion. The most commonly known method is based on the direction of harmonic power [5], which has been proven to provide unreliable results under specific conditions [6]. The general concept of the method described in the relevant IEC reports (IEC vector method) is based on the change of the magnitude of the disturbing voltage phasor before and after connection of an installation. The "harmonic vector method" (HVM) is based on the Thevenin (Norton) equivalent circuit model. Applying the superposition principle, it could be possible to quantify the contribution of customer and network on the harmonic voltage phasor $[7,8]$.

In this paper a medium-sized PV installation is studied with respect to different aspects of harmonic emission based on field measurements. This includes the summation of individual inverters, the impact of voltage distortion on harmonic currents and assessment of the impact of the PV installation to the supply voltage distortion based on the two methods mentioned above.

After a short description of the measurement framework, harmonic voltages and currents for individual PV inverters as well as the complete PV installation are presented. Prevailing harmonic phase angles and summation exponents are discussed. Individual harmonic currents are compared with the emission levels provided by the manufacturer in the PV inverter certificate as well as with limits provided for the whole PV installation by different standards. Furthermore, the network harmonic impedance measurements at the connection point of the $\mathrm{PV}$ installation are discussed with respect to the number of connected PV inverters. Based on the impedance measurements the results for IEC vector method and HVM method for contribution assessment are compared in section 6. Finally, some conclusions are provided.

\section{Measurement Framework}

\section{A. Measurement Setup}

The aim of the measurement is to characterize the harmonic behavior of individual PV inverters as well as of the entire PV installation. Figure 1 presents an overview of the network, the PV installation and the measurement locations. The PV installation is connected to the low voltage $(400 \mathrm{~V})$ side of a $630 \mathrm{kVA}$ distribution transformer, which supplies further commercial loads by other feeders.

The PV installation has a total rated power of $103 \mathrm{~kW}$ and consists of 16 single-phase PV inverters of two types. Type A (PVI_A) has a rated power of $3.3 \mathrm{~kW}$ (PVI_9). The remaining PV inverters belong to type B (PVI_B) and are rated at $6.65 \mathrm{~kW}$. It should be noted that the maximum power values observed for the PV inverters 


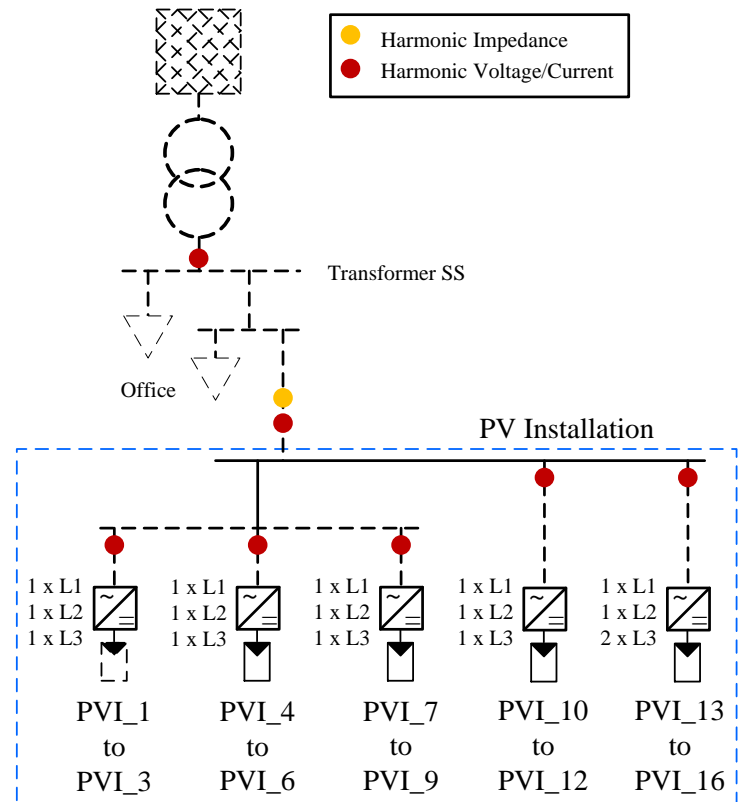

Fig. 1. General scheme of network and measurement locations

during the measurement interval are $2.15 \mathrm{~kW}$ for PVI_A and $4.2 \mathrm{~kW}$ for PVI_B.

Seven PQ instruments, which comply with IEC 61000-430 Ed.3 class A, were used for the measurements. Each current input channel of the PQ instruments was allocated to one PV inverter except for two PV inverters, which were both measured together with one input channel. An external synchronisation by GPS was applied to ensure time-synchronised measurements.

\section{B. Measurement Procedure}

The measurement is divided into two parts: an invasive and a non-invasive measurement. In the case of the noninvasive measurement the harmonics have been measured without any manipulation of PV inverter operating conditions for a period of two weeks. "Very-short-term" values $(3 \mathrm{sec})$ of harmonic current and voltage magnitudes and phase angles up to $50^{\text {th }}$ harmonic order are available for 8 days. Only data measured during the times, where PV inverters have been switched on is considered for the analysis unless it is explicitly stated different.

The invasive measurement has been performed during one day of the total measurement time. During this time the PV inverters have been switched off one after each other in order to study the harmonic emission as a function of the

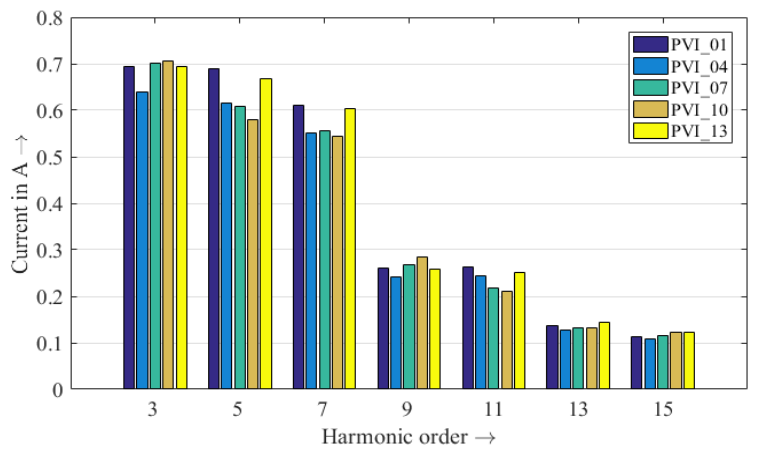

Fig. 3. Spectrum of the $99^{\text {th }}$ percentile of harmonic currents of individual PV inverters connected to phase L1
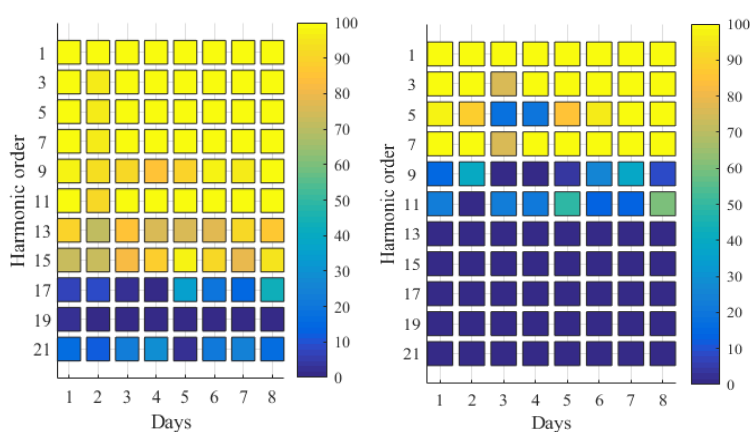

Fig. 2. Percentage of valid data for PVI_B (left) and PVI_A (right) during 8 days measurement

number of connected PV inverters as well as to assess the impact of the number of PV inverters on the frequencydependent network impedances.

\section{Data Validation}

All measured data were validated according to measurement accuracy and all data with an uncertainty higher than $10 \%$ has been removed from the data set. The percentage of remaining data is shown in Figure 2. For PVI_B as well as the PV installation nearly all of the odd harmonics up to $15^{\text {th }}$ order remain in the data set for further analyses. For PVI_A due to the lower rated power only odd harmonics up to $7^{\text {th }}$ order can be further processed.

\section{Analysis of Individual PV Inverters}

\section{A. Harmonic current spectrum}

Figure 3 shows the $99^{\text {th }}$ percentile of harmonic current magnitudes of the individual PV inverters connected to phase L1. $3^{\text {rd }}, 5^{\text {th }}$ and $7^{\text {th }}$ harmonic are the dominating harmonic currents with magnitudes between $0.5 \mathrm{~A}$ and $0.7 \mathrm{~A}$. Furthermore, a certain deviation in the harmonic current between the different PV inverters can be observed, even if they all belong to the same type (PVI_B).

\section{B. Daily Variation}

The harmonic currents of the individual PV inverters vary significantly during the day. This is mainly caused by the change in output power and the variation of the supply voltage distortion. Figure 4 illustrates this issue exemplarily for PVI_10 by box-whisker diagrams of harmonic currents and voltages for a sample cloudy and sunny day.
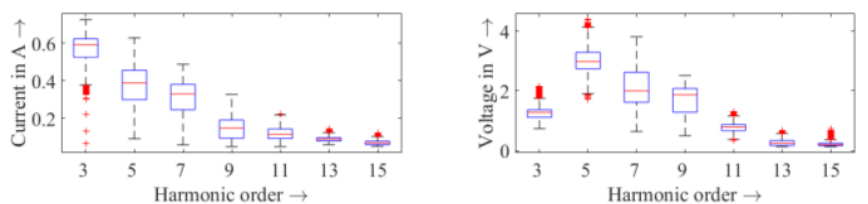

(a)
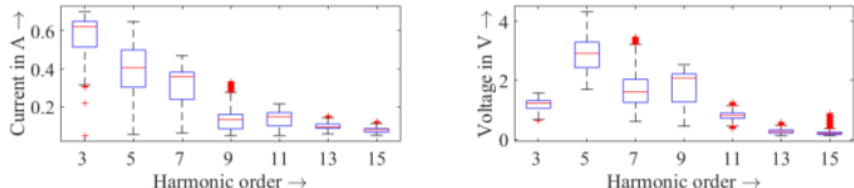

(b)

Fig. 4. Variation of voltage and current harmonics on a cloudy (a) and a sunny (b) day for PVI_10 

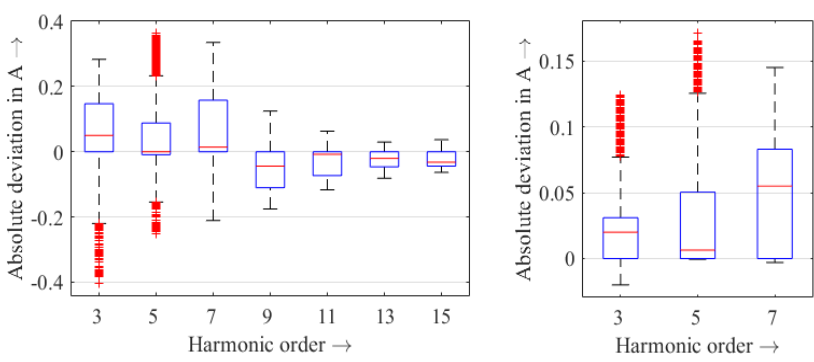

Fig. 5. Absolute deviation of the harmonic currents for PVI_A (right) and PVI_B (left)

\section{Comparison with Limits}

In Germany, a so-called unit certificate has to be provided by the manufacturer for each individual PV inverter. This certificate also includes harmonic currents. As these values have been obtained in the laboratory at different conditions (supply voltage distortion, network impedance) compared to the final connection point, they often differ from measurements in the field. Figure 5 shows absolute deviation of the harmonic currents for PVI_A and PVI_B from the limits provided by manufacturer. Both positive and negative deviations are possible. With increasing harmonic order the deviation decrease, which is a result of the decrease of the respective harmonic background voltage.

\section{Analysis of the PV Installation}

\section{A. Harmonic current spectrum}

Figure 6 shows the $99^{\text {th }}$ percentile of harmonic current spectrum of the entire PV installation. $3^{\text {rd }}, 5^{\text {th }}$ and $7^{\text {th }}$ harmonics are the dominating orders. Considerable unbalances between the phases can be observed for selected harmonic orders. However, with about 3.9 A total harmonic current (THC), which corresponds to about $2.6 \%$ total demand distortion (TDD), the harmonic currents are small.

\section{B. Summation Exponents}

Since the harmonic currents of the PV installation as well as the individual PV inverters have been measured, the summation exponent $\alpha$ can be determined by solving the following equation [9]:

$$
I_{h}=\sqrt[\alpha]{\sum_{i=1}^{n}\left|\underline{I}_{i, h}\right|^{\alpha}}
$$

where $\underline{I}_{i, h}$ is the $h^{\text {th }}$ harmonic current phasor of the individual PV inverter, $\mathrm{n}$ is total number of $\mathrm{PV}$ inverters

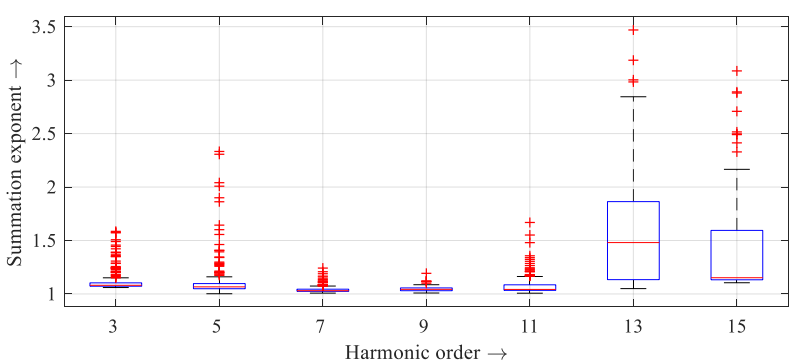

Fig. 7. Deviation of summation exponents

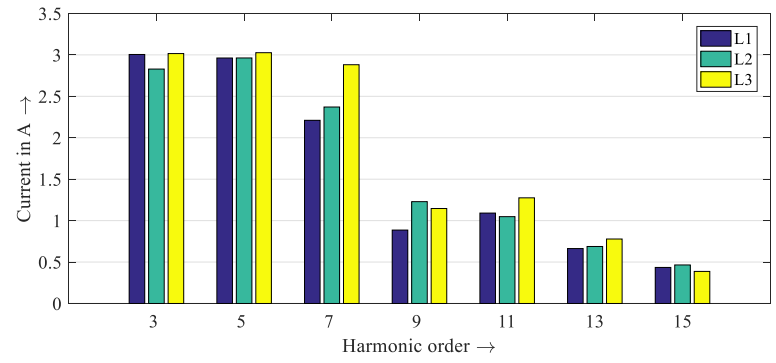

Fig. 6. Spectrum of the $99^{\text {th }}$ percentile of harmonic currents of the PV installation

and $I_{h}$ is the magnitude of the total $h^{\text {th }}$ harmonic current of the PV installation. Considering (1), it can be obviously seen that the summation exponent would approach infinity in case of a perfect cancellation between the current harmonics. Contrary it will amount 1 , if all individual currents are similar and sum up arithmetically. Figure 8 exemplarily presents the range of variation of summation exponents of phase L1. It shows that the odd harmonic currents of the individual PV inverters up to 11th order sum up almost arithmetically. This confirms the findings in [9] and suggests that standard summation exponents according to e.g. IEC 61000-3-14 should not be applied for devices with similar harmonic characteristics. The other phases behave similar.

\section{Comparison with Limits}

Individual harmonic emission limits $I_{\mathrm{h} \text { lim }}$ are calculated for the PV installation according to the German rule ARN 4105 [2]:

$$
I_{h \lim }=i_{h t a b} \cdot S_{s c} \cdot \sqrt[\alpha]{\frac{S_{i}}{S_{t o t}}}
$$

where $i_{\mathrm{h} \text { tab }}$ is a tabulated reference value, $\mathrm{S}_{\mathrm{sc}}$ is the short circuit power at the connection point, $S_{i}$ the rated power of the installation and $S_{\text {tot }}$ the total connection capacity of the node calculated based on the loading capacity of the supplying cable. Figure 8 exemplarily compares the calculated limits with the measured values of the PV installation at phase L1. It should be noted that no limit exists for $15^{\text {th }}$ harmonic current in the present version of the standard. As it can be clearly seen, all the limits are fulfilled. Similar results have been obtained for the other two phases.

With respect to IEEE 519-2014, the $99^{\text {th }}$ percentiles of the 3-second-values presented in Figure 6 are all below the respective limits for the $95^{\text {th }}$ percentile (10-minaverage). Therefore, the PV installation also complies fully with this standard.

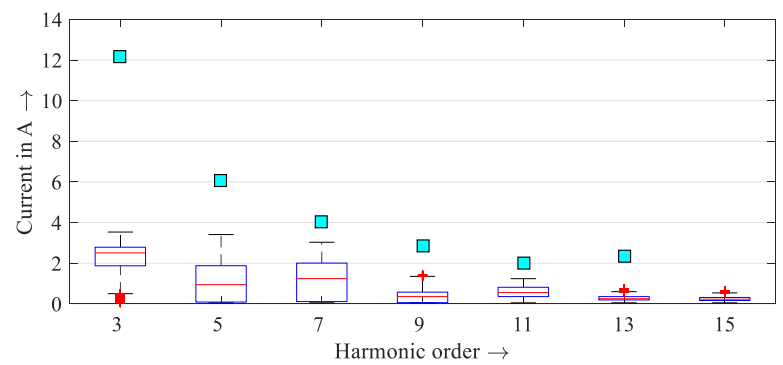

Fig. 8. Variation of harmonic currents of the PV installation (phase L1) and the corresponding emission limits 


\section{Prevailing Harmonic Current location}

In order to characterize the potential of cancellation with harmonic currents of other feeders/equipment (primary cancellation) or to calculate the impact of the harmonic currents to the respective supply voltage harmonics (secondary cancellation), prevailing phasors are calculated for the dominating current harmonic orders according to [10]. This reference provides also further details on primary and secondary cancellation. The prevailing harmonic current phasor $\underline{I}_{p r v, h}$ is determined as

$$
\underline{I}_{p r v, h}=I_{p r v, h} \angle \varphi_{p r v, h}
$$

Where $I_{p r v, h}$ represents the RMS value of the individual magnitudes, and $\varphi_{p r v, h}$ is the phase angle of the sum of individual harmonic phasors for a considered time interval. In order to determine the significance of the prevailing harmonic phasors, the level of prevalence is calculated for the considered data set. Only in case the prevailing ratio is higher than 0.8 , a prevailing harmonic phasor is meaningful and should be reported.

The results of harmonic current prevailing phasors are summarised in Table 1. By taking the four prevalence ranges introduced in [10], it can be seen that prevailing harmonic current phasors of orders 3,7 and 11 have a high level of prevalence. In contrast, harmonic current phasors of order 9, 13 (except L2) and 15 (except L3) are too dispersed and no prevailing direction of harmonic emission exists.

Table 1. Prevailing harmonic current phasors (green: high prevalence; yellow: medium prevalence; red: low

\begin{tabular}{|c|c|c|c|}
\multicolumn{4}{c}{ prevalence; white: no prevalence) } \\
\hline $\begin{array}{c}\text { Harmonic } \\
\text { order }\end{array}$ & L1 & L2 & L3 \\
\hline 3 & $1.96 \mathrm{~A} \angle 95.2^{\circ}$ & $1.84 \angle 90.8^{\circ}$ & $1.90 \angle 92.0^{\circ}$ \\
\hline 5 & $1.38 \angle 342.8^{\circ}$ & $1.37 \angle 333.6^{\circ}$ & $1.41 \angle 350.5^{\circ}$ \\
\hline 7 & $1.48 \angle 5.6^{\circ}$ & $1.52 \angle 5.1^{\circ}$ & $1.81 \angle 14.0^{\circ}$ \\
\hline 9 & $\mathrm{NA}$ & $\mathrm{NA}$ & $\mathrm{NA}$ \\
\hline 11 & $0.53 \angle 329.8^{\circ}$ & $0.47 \angle 345.1^{\circ}$ & $0.58 \angle 337.1^{\circ}$ \\
\hline 13 & $\mathrm{NA}$ & $0.26 \angle 307.1^{\circ}$ & $\mathrm{NA}$ \\
\hline 15 & $\mathrm{NA}$ & $\mathrm{NA}$ & $0.18 \angle 287.6^{\circ}$ \\
\hline
\end{tabular}

In typical residential networks $3^{\text {rd }}$ harmonic current is located at about $200^{\circ}$ and $5^{\text {th }}$ harmonic current at about $325^{\circ}$ [10]. Comparing these directions with the emission of the PV installation identifies almost no potential of primary cancellation for $5^{\text {th }}$ harmonic (almost similar direction), but a certain potential for primary cancellation for $3^{\text {rd }}$ harmonic current (phase angle difference of about $100^{\circ}$.

\section{E. Correlation of Harmonics with Active Power}

The dependency of harmonic currents on the output power of the PV installation is studied based on the invasive measurement, where PV inverters have been switched off one after each other (cf. section 2.B). Figure 9 confirms that the harmonic current magnitude of the PV installation clearly increases with increasing number of active PV inverters. In contrast, harmonic voltage magnitudes slightly decrease except for $5^{\text {th }}$ harmonic and no adverse effect of the PV installation on the harmonic voltages, at least during the experiment, could be observed.
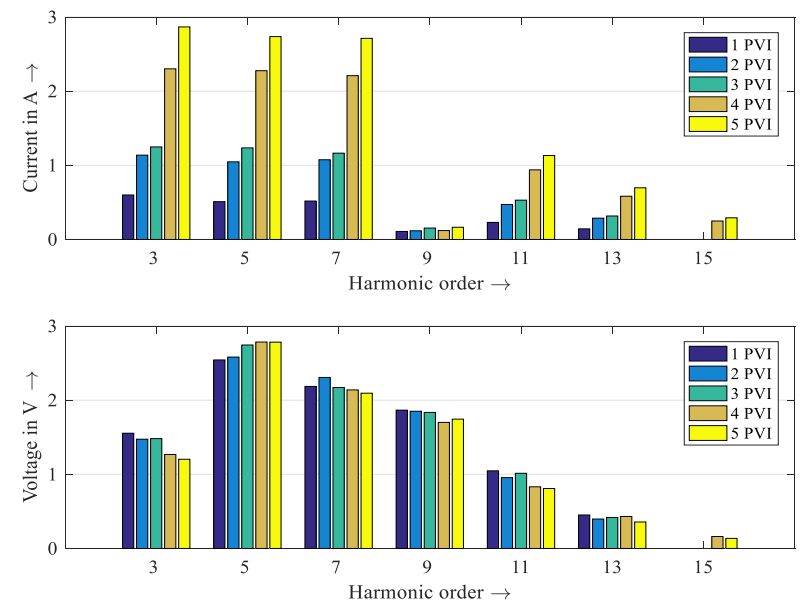

Fig. 9. Spectrum of $99^{\text {th }}$ percentile of harmonic currents and voltages at phase L3 of the PV installation

Figure 10 studies the opposing tendency of $3^{\text {rd }}$ harmonic current and voltage in more detail using polar plot representations. Hence, the harmonic current increases almost linear. The harmonic voltage drop caused by this current at the network impedance has a favourable location with respect to the background harmonic voltage. Consequently, the increasing harmonic current shifts and reduces the harmonic voltages at the connection point of the PV installation (secondary cancellation).

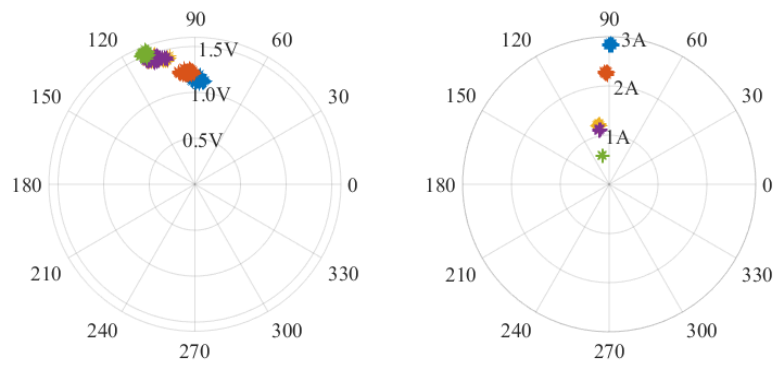

Fig. 10. Polar plot of the $3^{\text {rd }}$ harmonic voltage (left) and current (right) at different operating conditions of the PV installation

\section{Harmonic Impedance}

Harmonic impedance has been measured during the invasive measurements using an impedance measurement system developed by the authors and described in [11]. Figure 11 shows the magnitude and the phase angle of the frequency-dependent impedance in phase L1 for different numbers of connected PV inverters. A resonance at approximately $800 \mathrm{~Hz}$ can be observed for all switching states, even in case no PV inverter is connected. Consequently, it is at least partly determined by other consumers. Without any PV inverter connected, the harmonic impedance increases after the resonance almost linear with the frequency. As the impedance angle remains clearly inductive this impedance is dominated by the network components. If the first PV inverter is connected, two further resonances are observed at about $3 \mathrm{kHz}$. These resonances are caused by the grid-side filter circuits of the PV inverters, which include shunt capacitances. The reduced phase angle within the resonance (compared to the impedance without PV 

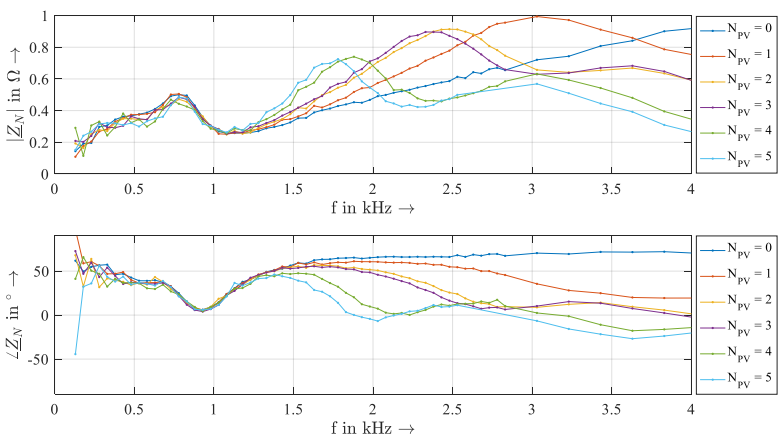

Fig. 11. Harmonic impedance for different number of $\mathrm{PV}$ inverters

inverters) as well as the clear dependency of the resonance frequency on the number of PV inverters confirms this assumption. At higher frequencies, the PV installation can act as filter.

The (total) harmonic impedance $\underline{Z}_{\mathrm{hg}}$ at the connection point of the installation (POC) is composed by the parallel circuit of the network harmonic impedance $\underline{Z}_{\mathrm{hn}}$ (sum of impedances on the grid-side of the POC) and the customer harmonic impedance $\underline{Z}_{\mathrm{hc}}$ (impedance of the PV installation on the customer-side of the POC). As network harmonic impedance during the invasive measurement can be assumed to be almost constant, the customer harmonic impedance of the PV installation (all PVI connected) can be calculated based on network harmonic impedance (no PVI connected) and total harmonic impedance (all PVI connected) according to

$$
\underline{Z}_{h c}=\frac{\underline{Z}_{h n} \cdot \underline{Z}_{h t}}{\underline{Z}_{h n}-\underline{Z}_{h t}}
$$

Figure 12 presents the respective customer and network harmonic impedance characteristics.

It should be noted that due to the high short circuit power at the point of connection (about $8 \mathrm{MVA}$ ) the impedance measurements have a relatively high uncertainty, particularly at lower frequencies. This is reflected by the "noise" in Figure 11 and impacts also the accuracy of the customer impedance. However, the results can still serve as first indicator.

\section{Harmonic Contribution Assessment}

This section presents the application of two different methods for the assessment of harmonic contributions of customer installations, namely the IEC vector method and the harmonic vector method (HVM). Further details on the methods can e.g. be found in [7]. Both methods require knowledge of voltage and current harmonic phasors at the point of common coupling (PCC) as well as the network harmonic impedance. In addition, the HVM needs to know impedance of the customer installation as well. All required input parameters are available from the measurements described in section 2 and 5 .

\section{A. IEC Method}

The harmonic emission phasor of a distorted installation is the difference between the voltage harmonic before and after connection of the installation. Figure 13 presents the phasor diagram of the harmonic voltages.
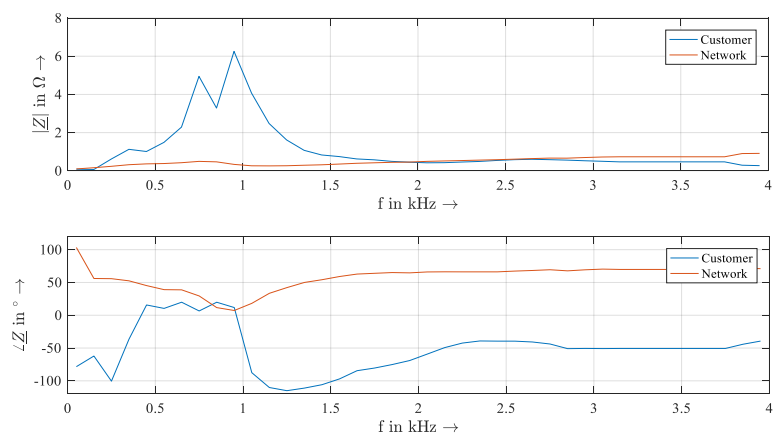

Fig. 12. Harmonic impedance of the customer and network

$\underline{U}_{h b g}, \underline{U}_{h P C C}$, and $\underline{U}_{h i}$ represent the voltage harmonic before connection of an installation, voltage harmonic after connection of an installation and the disturbing voltage vector, respectively.

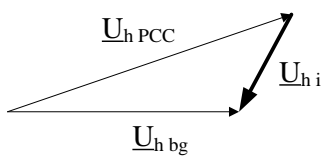

Fig. 13. Illustration of the voltage emission phasors

The background harmonic voltage can be calculated by:

$$
\underline{U}_{h b g}=\underline{U}_{h P C C}+\underline{Z}_{h} \cdot \underline{I}_{h P C C}
$$

According to IEC an installation emits harmonics, if the magnitude of harmonic voltage at PCC, $U_{h P C C}$ is larger than the magnitude of the background harmonic voltage, $U_{h b g}$ :

$$
U_{h P C C}-U_{h b g}>0
$$

\section{B. Harmonic Vector Method}

The harmonic vector method (HVM) is based on the Thevenin (Norton) equivalent circuit models of the customer and utility (network) side [7,8]. Applying the superposition principle, the harmonic contribution of customer and network side on the harmonic voltages at PCC can be separated by phasor projection like presented in Figure 14.

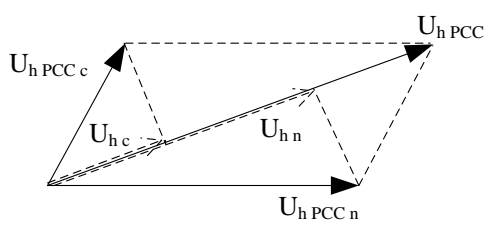

Fig. 14. Illustration of scalar contribution of network and customer harmonic voltage phasors.

\section{Results and Discussion}

Both methods have been applied to the PV installation on the measurements of a sunny day and the time interval when the PV installation has been active. The PCC corresponds to the POC of the PV installation. Figure 15 exemplarily illustrates the different voltage components of both methods for $3^{\text {rd }}$ and $5^{\text {th }}$ harmonic in phase L2. With respect to IEC method for the $3^{\text {rd }}$ harmonic (Figure 15 , left) the difference according to (6) is negative in most of the time, which means the PV installation reduces the $3^{\text {rd }}$ harmonic voltage at the POC. This agrees 

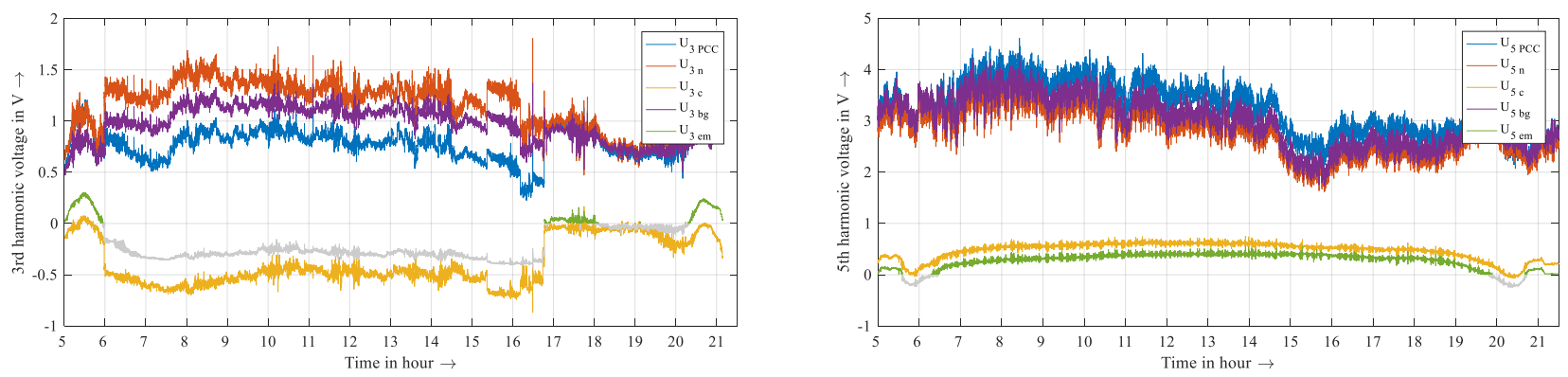

Fig. 15. Comparison of the harmonic voltage components for IEC and HVM method for $3^{\text {rd }}$ harmonic (left) and $5^{\text {th }}$ harmonic (right)

with the conclusion drawn in section 4.E. The HVM provides similar results, however during most of the time a slight offset can be observed. In contrary, for $5^{\text {th }}$ harmonic (Figure 15, right) most of the time the difference for IEC method is positive, which means that the PV installation emits harmonics and increases the harmonic voltage at the POC. The same result is obtained for the HVM. A slight difference exists between both methods, but now opposite than for the $3^{\text {rd }}$ harmonic. Table 2 summarizes the time share of the considered interval when the PV installation emits harmonics. An increasing effect can be observed for $5^{\text {th }}$ and $7^{\text {th }}$ voltage harmonic. However, this has to be compared with the permissible emission limits in order to finally evaluate the grid compliance of the PV installation. The partly high differences in the table are caused by the differences in the evaluated voltage component $\left(\mathrm{U}_{\mathrm{hem}}\right.$ for IEC method, $\mathrm{U}_{\mathrm{hc}}$ for $\mathrm{HVM}$ ) and possible reasons have to be further explored.

Table 2. Ratio of time that the PV installation has harmonic emission

\begin{tabular}{|c|c|c|c|c|c|c|c|}
\hline & 3 & 5 & 7 & 9 & 11 & 13 & 15 \\
\hline IEC & 0.13 & 0.93 & 0.57 & 0.39 & 0.01 & 0.23 & 0.41 \\
\hline HVM & 0.02 & 0.99 & 1.00 & 0.94 & 0.55 & 0.50 & 0.65 \\
\hline
\end{tabular}

\section{Conclusion}

The paper studies the harmonic emission characteristics of a medium-sized PV installation. The comparison of the harmonic currents of the individual PV inverters with the values provided by manufacturer shows a significant difference for some harmonics. Consequently, care should be taken, if the values are used to calculate the emission of a PV installation in planning stage. The harmonic currents of the whole PV installation meet the limits both of the German standard VDE AR-N 4105 and the IEEE standard 519-2014. The analysis of the location of harmonic currents as well as the harmonic voltages in dependence on output power identifies a cancellation for the $3^{\text {rd }}$ harmonic. This confirms that PV installations do not necessarily have only an adverse effect on the supply voltage distortion. In case harmonic current emission limits are exceeded, the impact on the respective voltage harmonics should be always assessed before mitigation measures are considered. IEC vector method and harmonic vector method provide useful tools for the assessment of the true impact of the PV installation on supply voltage harmonics. The results of this study, in particular the application of the assessment methods serve as input for the CIGRE working group $\mathrm{C} 4.42$, which deals with the identification of customer contribution with respect to low order harmonics.

\section{Acknowledgement}

This work was supported by the German Federal Ministry for Economic Affairs and Energy (BMWi) (project NetzHarmonie, FKZ: 0325757K).

\section{References}

[1] Electromagnetic compatibility (EMC) - Part 3-14: Assessment of emission limits for harmonics, interharmonics, voltage fluctuations and unbalance for the connection of disturbing installations to $\mathrm{LV}$ power systems, IEC TR Std. 61000-3-14, 2011.

[2] Generators connected to the low-voltage distribution network - Technical requirements for the connection to and parallel operation with low-voltage distribution networks, VDE-AR-N 4105, 2011.

[3] M. Aiello, A. Cataliotti, S. Favuzza, G. Graditi, "Theoretical and experimental comparison of total harmonic distortion factors for the evaluation of harmonic and interharmonic pollution of grid-connected photovoltaic systems", IEEE Transactions on Power Delivery, 21(3), 2006, pp. 1390-1397.

[4] R. Arghandeh, A. Onen, J. Jung, D. Cheng, R.P. Broadwater V. Centeno, "Phasor-based assessment for harmonic sources in distribution networks", Electric Power Systems Research, 116, 2014, pp. 94-105.

[5] L. Cristaldi and A. Ferrero, "Harmonic power flow analysis for the measurement of the electric power quality," IEEE Trans. Instrum. Meas., vol. 44, 1995, pp. 683-685.

[6] Xu, W., Liu, X., Liu, Y. "An investigation on the validity of power-direction method for harmonic source determination', IEEE Trans. Power Deliv., 2003, 18, (1), pp. 214-219.

[7] A. Špelko, B. Blažič, I. Papič, M. Pourarab, J. Meyer, X. $\mathrm{Xu}$ and S. Z. Djokic, "CIGRE/CIRED JWG C.4.42: Overview of Common Methods for Assessment of Harmonic Contribution from Customer Installation," in $12^{\text {th }}$ IEEE PES PowerTech Conference, Manchester, 2017.

[8] T. Pfajfar, B. Blažič and I. Papič, "Methods for estimating customer voltage harmonic emission levels," ICHQP 2008: 13th International Conference on Harmonics and Quality of Power, Wollongong, Australia, 2008, pp. 1-6.

[9] A. Varatharajan, S. Schoettke, J. Meyer, and A. Abart, "Harmonic Emission of Large PV Installations Case Study of a 1 MW Solar Campus," in International conference on Renewable Energies and Power Quality (ICREPQ'14), 2014, pp. 1-6.

[10] J. Meyer, A. Blanco Castaneda, M. Domagk, P. Schegner, "Assessment of Prevailing Harmonic Current Emission in Public Low Voltage Networks," in IEEE Transactions on Power Delivery, in press.

[11] R. Stiegler, J. Meyer, P. Schegner and D. Chakravorty, "Measurement of network harmonic impedance in presence of electronic equipment", IEEE International Workshop on Applied Measurements for Power Systems (AMPS), September 2015, Aachen, Germany. 